\title{
The Flux of Particles to the Deep Sea: Methods, MEASUREMENTS, AND MECHANISMS
}

\author{
By Wilford D. Gardner
}

\section{"D}

ON'T PARTICLES JUST sink?" This query was raised by a non-oceanographer upon hearing about a class I teach at Texas A\&M on the transport of particles from the sea surface to the sea floor. Although many particles do sink, their pathway to the sea floor is seldom direct, and most of them never complete the journey.

Two reasons for measuring the flux of particles are 1) to quantify the exchange rates in biogeochemical cycles involving important compounds (e.g., establishing realistic budgets of carbon, including sources and sinks, is especially important for determining the uptake of $\mathrm{CO}_{2}$ in the ocean, which is pertinent to the issue of global warming), and 2) to determine which particles complete the journey to the sea floor-information that will aid in interpreting the paleoceanographic record of climate history. In covering these issues, I will briefly discuss the evolution of different approaches and calculations made to determine fluxes, beginning with particle models, then sediment traps, aggregate abundance, ${ }^{234} \mathrm{Th}$ deficiency, and ending with carbon budgets developed in programs of the Joint Global Ocean Flux Study (JGOFS). Then I'll discuss the "mixed-layer pump" as a mechanism of exporting particles from surface waters.

Nearly all particles in surface waters of the open ocean are of biogenic origin. River-borne particles mostly settle out in estuaries or on the shelf; aeolian dust entering the ocean is quickly exported via biological packaging. Most phytoplankton are too small to settle very rapidly by themselves (one to a few

Wilford D. Gardner, Department of Oceanography, Texas A\&M University, College Station, TX 77843-3146, USA. $\mathrm{m} / \mathrm{d}$ ), but they can become aggregated and sink out of the surface waters faster. Phytoplankton can be ingested by zooplankton, whose by-products are released by respiration and excretion. The resulting fecal pellets can settle directly or become incorporated into aggregates and sink (Fig. 1). Zooplankton can also migrate vertically, sometimes several hundred meters, carrying downward carbon and other materials consumed or metabolized at the surface while respiration and excretion of carbon products continue. This is termed active transport. One by-product of biogenic processes is dissolved organic matter (DOM), which doesn't settle by gravity but can diffuse or be mixed vertically and laterally in the water column.

\section{Particle Fluxes}

Before sediment traps were widely used, the determination of particle fluxes in the ocean centered on measuring the size and abundance of particles, estimating their density, and calculating a flux based on Stokesian settling velocities (Lal and Lerman, 1973). McCave (1975) nicely articulated what was becoming obvious by demonstrating that the largest particles were responsible for most of the particle flux. He first modeled the size distribution of particles based on a wide spectrum of assumptions, then calculated their volume, assigned densities based on published values, and determined their mass, Stokesian settling velocity, and finally the resulting mass flux. Regardless of the extremes of the size distribution assumed for the particles, the largest particles always dominated the flux in the model. For example, the upper two size ranges-128-512 $\mu \mathrm{m}$-accounted for $4-28 \%$ of the mass, but $47-89 \%$ of the flux. Since 1975, we've learned two addi- tional points that have modified our concepts about particle fluxes. One is that large aggregates $(>500 \mu \mathrm{m})$ are far more abundant than had been assumed by McCave (1975), further emphasizing the importance of large particles. The second is that the bulk density of aggregates can be much smaller than assumed by McCave, to the point where particles could become neutrally buoyant. Therefore we really have to think about "rapidly settling" particles rather than "large" particles as dominating particle flux.

With the realization that the large particles (e.g., $>63 \mu \mathrm{m}$ ) must be responsible for most of the mass flux in the ocean, the focus of a few researchers shifted to measuring larger volumes of water by in situ pumping to obtain these rare particles to estimate fluxes and analyze particle composition (Bishop et al., 1980). However, because of advances in the reliability and economy of mooring technology,

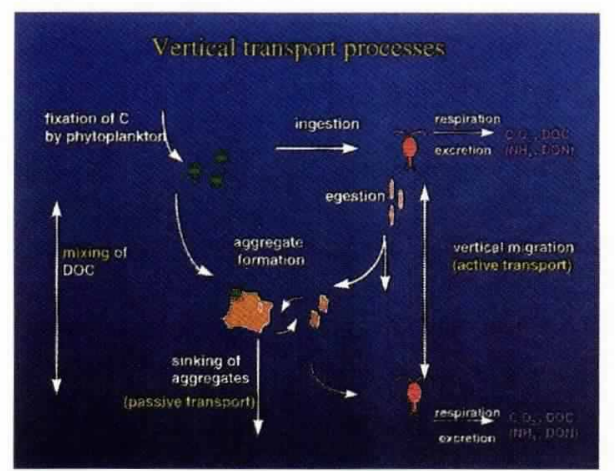

Fig. 1: Processes by which carbon in the ocean is fixed and transported vertically [D. Steinberg, Bermuda Biological Station for Research (BBSR)]. Sediment traps can be used to collect passively sinking particles, but not vertically mixed dissolved organic carbon (DOC) or carbon transported by migrating zooplankton. 
most investigators turned to sediment traps to let the particles come to them with the hope that settling particles would be separated naturally from slowly settling or neutrally buoyant particles (commonly referred to as "suspended" particles; a misnomer since no suspending force is involved). Sediment traps have ranged in shape and size from $7-\mathrm{cm}$ cylindrical tubes to funnels more than 1 $m$ in diameter with sample changers at the bottom for time-series measurements. A discussion of the methods and challenges in using sediment traps can be found in US GOFS Report No. 10 (1989). A more recent report (from the First International JGOFS Symposium in Villefranche) on the use of floating traps in the upper $200 \mathrm{~m}$ is available at http://wwwocean.tamu.edu/JGOFS/contents.html.

\section{Aggregates}

One of the early observations about the material collected with sediment traps was the abundance of large fecal pellets. The "fecal pellet express" initially received considerable attention, but then came the realization that trap samples also contained substantial amounts of amorphous material that was assumed to be "marine snow"; there was often more amorphous material than fecal pellets. The question was: How do you sample or analyze these amorphous particles since their integrity and shape were not maintained in the trap? Scuba divers photographed marine snow in situ (Shanks and Trent, 1980), and later Honjo et al. (1984) built a camera system that could image marine snow at all depths in a quantifiable way. The principle was to illuminate a thin slab of water using a strobe light and photograph the illuminated particles in the slab. The number, size, and shape of the particles are determined using image analysis software. Volume can be estimated with assumptions about sphericity; mass and settling velocity can be estimated with assumptions about density and Stokes' Law, or with empirical relationships. Similar cameras have been developed at several institutions, including at Texas A\&M, where our latest system has two video cameras with different focal lengths and separate strobe lights to cover a wider range of particle sizes, starting at $250 \mu \mathrm{m}$. To this large-aggregate profiling system (LAPS) we have added numerous other optical instruments to monitor the abundance and characteristics of particles from micrometers to millimeters in size, creating a particle and optics profiling system (POPS).

A profile with LAPS off Cape Hatteras made by Ian Walsh during the U.S. Department of Energy Ocean Margins Program showed a peak in beam attenuation (caused by particles $<20 \mu \mathrm{m}$ ) at $\sim 40$ $\mathrm{m}$, below which the signal dropped to a fairly constant value from $75 \mathrm{~m}$ down to $600 \mathrm{~m}$. The abundance of large aggregates similarly exhibited a peak at $40 \mathrm{~m}$, with the number of aggregates decreasing to a minimum at $180 \mathrm{~m}$. In contrast to the constant concentration of small particles, there were several layers of aggregates between 200 and $600 \mathrm{~m}$, the deepest peaks being as large as the surface peak. We assume these deeper layers resulted from advection of aggregates off the continental slope, but presence of this material was not detected with the transmissometer (Gardner and Walsh, 1990).

From the data on number and size of aggregates per liter, we can make assumptions about their density and settling velocity and provide an estimate of the downward mass flux of particles. For example, in the JGOFS North Atlantic Bloom Experiment (NABE), we made several profiles with the LAPS system during the time when a floating sediment trap array was collecting particles (Martin et al., 1993). Our estimates of mass flux were very similar to the trap-measured fluxes (Fig. 2). Note that the flux at $1,500 \mathrm{~m}$ measured with floating Moss Landing Marine Lab (MLML) traps was almost identical to the flux measured with a moored trap over the same time period (Honjo and Manganini, 1993).

Vernon Asper advanced the study of aggregates by developing a system to measure their settling velocity in situ (Diercks and Asper, 1997). It consists of tubes that act as sediment traps into which particles settle or are advected. Once particles penetrate below the turbulence zone, they settle in quiescent Plexiglas chambers where time-lapse photography allows the measurement of the sinking velocity of aggregates. Asper (personal communication) deployed this instrument during two field seasons in the Ross Sea and found that most aggregates were between 1 and $3 \mathrm{~mm}$ in size and their settling speeds ranged from a few meters to almost $250 \mathrm{~m} / \mathrm{d}$. Unfortu- nately, in that particular environment there wasn't a good correlation between the aggregate diameter and settling speed. So it's not always going to be simple to calculate a flux based simply on the size of aggregates, although we can at least infer something about sources and activity of aggregates based on knowledge of their size and distribution in the water column.

\section{${ }^{234}$ Th Deficiency Proxy for Carbon Fluxes}

Although sediment traps are valuable tools in measuring particle fluxes, their accuracy in surface waters has been questioned, and there are situations when time and space considerations make them difficult to use. It would be helpful if we could devise a proxy for fluxes of carbon or any material in the ocean. One proxy that is being used is ${ }^{234} \mathrm{Th}$. The method is based on the radioisotope ${ }^{238} \mathrm{U}$, which is well mixed in the ocean because it does not react chemically and does not adhere to particles, whereas its daughter product, ${ }^{234} \mathrm{Th}$ quickly adheres to a particle of any size. As these particles sink, either as individual particles or as aggregates, they remove ${ }^{234} \mathrm{Th}$ from surface waters. This creates a disequilibrium between the ${ }^{234} \mathrm{Th}$ and ${ }^{238} \mathrm{U}$ concentrations. A flux of ${ }^{234} \mathrm{Th}$

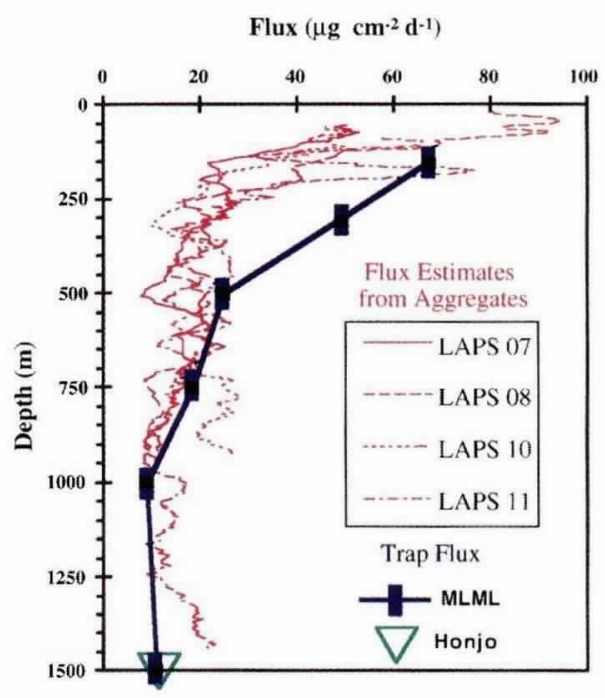

Fig. 2: Mass fluxes measured with floating sediment traps (J. Martin, Moss Landing Marine Laboratory), moored traps (S. Honjo, WHOI) and fluxes estimated from aggregate abundance and size measurements using our Large Aggregate Profiling System (LAPS) and settling velocity estimates (Walsh and Gardner, 1994). 
$\left(\mathrm{F}_{\mathrm{Th}}\right)$ out of the zone of disequilibrium $(\sim 100 \mathrm{~m})$, can be obtained from $\mathrm{F}_{\mathrm{Th}}=$ $\lambda_{\mathrm{Th}} * \int(\mathrm{U}-\mathrm{Th}) * \mathrm{dz}$, where $\lambda_{\mathrm{Th}}$ is the decay constant of ${ }^{234} \mathrm{Th}$.

Although the flux of ${ }^{234} \mathrm{Th}$ is not of particular interest to the JGOFS program, the flux of carbon is. Thus the flux of ${ }^{234} \mathrm{Th}$ is an important measurement if there is a relationship between carbon and ${ }^{234} \mathrm{Th}$. The critical information to know accurately is the $\mathrm{C} /{ }^{234} \mathrm{Th}$ ratio of the sinking particles, since that ratio times the flux of ${ }^{234} \mathrm{Th}$ should yield the flux of carbon; i.e., $\mathrm{F}_{\mathrm{C}}=\mathrm{F}_{\mathrm{Th}} *(\mathrm{C} /$ $\left.{ }^{234} \mathrm{Th}\right)$. Buesseler et al. (1994, 1995), Murray et al. (1996), and others have made measurements of ${ }^{234} \mathrm{Th}$ deficit and compared them with trap carbon fluxes with varying results. The $\mathrm{C} /{ }^{234} \mathrm{Th}$ ratio has been determined in two ways: from material collected in traps and from large-volume in situ filtration samples. Not surprisingly, ratios from the traps and filtration samples give different results since the filtered particles include both settling and neutrally buoyant particles, and the $\mathrm{C} /{ }^{234} \mathrm{Th}$ ratios from either source can change substantially during an active bloom with both time and depth (Table 1).

The ${ }^{234} \mathrm{Th}$ deficit method is not without its critics because of the uncertainty in the $\mathrm{C}^{234} \mathrm{Th}$ ratio and because the traps integrate the flux of particles over the time of their deployment, whereas the ${ }^{234} \mathrm{Th}$ method integrates roughly over a half-life of the radionuclide $-24 \mathrm{~d}$ in this case. Error bars are also large in areas where fluxes are small (Buesseler et al., 1994). Another limitation is that flux estimates based on the ${ }^{234} \mathrm{Th}$ method apply only to the depth over which a deficiency in ${ }^{234} \mathrm{Th}$ exists - on the order of $100 \mathrm{~m}$. More information is also needed on whether ${ }^{234} \mathrm{Th}$ is partitioned differently on carbon associated with siliceous versus carbonate organisms, etc.

Table 1

Ratio of particulate organic carbon to ${ }^{234} \mathrm{Th}$ from in situ filtration and from sediment traps deployed at two depths over three time periods in the North Atlantic Bloom Experiment.

\begin{tabular}{|c|c|c|c|}
\hline Deployment & Depth, m & Filter $\mathrm{POC}^{234} \mathrm{Th}, \mu \mathrm{mol} \mathrm{dpm}^{-1}$ & Trap $\mathrm{POCl}^{234} \mathrm{Th}, \mu \mathrm{mol} \mathrm{dpm}^{-1}$ \\
\hline \multirow[t]{2}{*}{1} & 150 & 15.0 & 3.9 \\
\hline & 300 & 10.6 & 2.4 \\
\hline \multirow[t]{2}{*}{2} & 150 & 11.8 & 5.9 \\
\hline & 300 & 8.0 & 3.6 \\
\hline \multirow[t]{2}{*}{3} & 150 & 8.9 & 7.4 \\
\hline & 300 & 7.9 & 4.0 \\
\hline
\end{tabular}

Data from Buesseler et al. (1992).
Results from a JGOFS program in the Equatorial Pacific (EqPac) using either sediment trap data or the ${ }^{234} \mathrm{Th}$ proxy method demonstrate that rapid recycling of carbon occurs in the ocean (Fig. 3). Primary production in a particular area and time of the EqPac program was 75 mmol m $\mathrm{m}^{-2} \mathrm{~d}^{-1}$. Taking this value as $100 \%$, let us examine fluxes as a percentage of primary production. The export from the euphotic zone as measured using traps between 100 and $200 \mathrm{~m}$ indicated that only $4-21 \%$ of the carbon produced at the surface was exported to that depth. Based on the ${ }^{234} \mathrm{Th}$ method, carbon export was between 3 and 5\%. Murray et al. (1996) used ${ }^{234} \mathrm{Th}$ data from the traps to correct the trap fluxes after which the trap fluxes were much closer to ${ }^{234} \mathrm{Th}$ based fluxes, although that approach is the sea floor indicate that only $0.4 \%$ of the organic carbon produced at the surface reached the sea floor, and only $1 \%$ of what reached the sea floor was actually preserved in the sediments. The major point was that on the order of $90 \%$ of the organic carbon produced in surface waters was recycled, not exported. Very little of the carbon fixed in surface waters escapes below the surface, much less to the sea floor.

Buesseler (personal communication) has plotted all carbon flux data based on the ${ }^{234} \mathrm{Th}$ deficiency method versus the primary production at each site and found that generally only $\sim 10 \%$ of the primary production is exported from the surface waters. Some noticeable exceptions occur in high latitudes and during bloom periods, when substantially more of the carbon is removed from surface waters.

\section{Mass Balance Models}

The next advance in calculating fluxes in the ocean was to calculate mass balsomewhat circular. Traps moored near

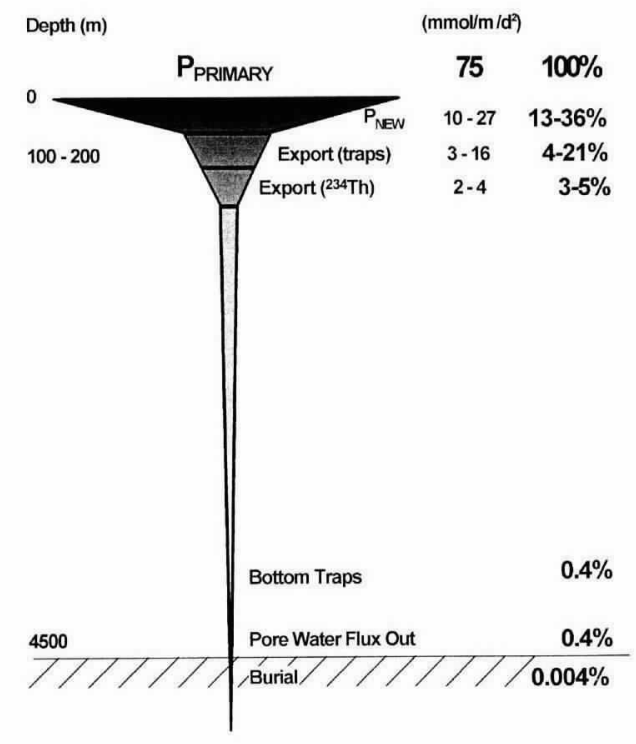

Fig. 3: Primary production and organic carbon flux at different depths using several methods of rate measurements during EqPac (modified from EqPac benthic group). Only a fraction of the carbon produced at the surface is exported below the euphotic zone, and only a trace of that carbon reaches the sea floor.

ances of carbon or oxygen. The JGOFS programs have measured enough parameters in time and space to begin making mass carbon budgets as a check on fluxes calculated with different methods. Michaels et al. (1994) found a three-fold carbon imbalance at the Bermuda Atlantic Time Series site (BATS) that is still being investigated, but inclusion of recent data on active transport by migrators has narrowed the gap to a factor of 2.4 (Steinberg et al., 1997). Conversely, carbon fluxes measured with sediment traps $\left(0.9 \pm 0.3\right.$ mole $\left.\mathrm{C} \mathrm{m}^{-2} \mathrm{y}^{-1}\right)(\mathrm{Karl}$ et al., 1996) at the Hawaii Ocean Time-Series (HOT) match very well the carbon flux calculated from an $\mathrm{O}_{2}$ mass balance model $\left(1 \pm 0.5 \mathrm{~mol} \mathrm{C} \mathrm{m}^{-2} \mathrm{y}^{-1}\right)$ (Emerson et al., 1995). See the Villefranche report at the internet URL given earlier for further discussion.

Organic carbon in the ocean can settle as particles, be carried down by zooplankton transport, be remineralized and mixed downward or advected laterally as DOM, or exhaled as $\mathrm{CO}_{2}$ from the sea surface. Because the focus of this paper 
is to examine the flux of particles in the ocean, the question is: "What percentage of the carbon entering the system leaves in the form of settling particles?" A compilation of data from several sources for JGOFS studies in the North Atlantic, Equatorial Pacific, and BATS suggests that the export of carbon from the euphotic zone by particle flux ranges from $\sim 50$ to $80 \%$. Thus, despite all of the legitimate interest in dissolved organic carbon in recent years, and the fact that $90 \%$ of the carbon produced in surface waters is remineralized there, carbon export is generally dominated by the flux of particulate carbon. Other elements, compounds, and plankton skeletons settling out of surface waters may remineralize more or less rapidly than carbon. Their rate of survival to the deep ocean or sea floor is important in determining biogeochemical cycles and in preserving paleoceanographic information, so that accurate determination of their fluxes is also important and requires the use of traps for collection.

\section{Mixed-Layer Pump: A Mechanism for Removing Particles from Surface Waters}

As oceanographers have investigated the complicated dynamics of the upper ocean in the last 10-20 y, there has been an increased focus on understanding the dynamic interaction among biology, physics, and optics. The rates of biological processes obviously rely on the availability of nutrients and light. The dynamics of the surface mixed layer of the ocean play a crucial role in controlling both the upward mixing of nutrients and the period of time plankton spend at different light levels. Mixed layer depth (MLD) varies not only seasonally, but on daily timescales with solar heating. nocturnal convective cooling, wind mixing, and subducting water masses. The MLD generally decreases during the day and increases at night, depending on the net effect of environmental factors. Particles produced in a thin surface layer during the day are mixed downward as the MLD thickens at a rate faster than the settling velocity of individual phytoplankton. Particles that remain below the MLD at night can settle in a zone of greatly diminished mixing, and rapidly settling particles can escape reincorporation into the ML the following night. This daily variation in the depth of mixing creates a "mixed-layer pump" that is an important mechanism of exchange between surface waters and the deep ocean.

What is the definition of the mixed layer? For plotting global data, Levitus (1982) defined the mixed layer as being the depth at which the density increased by $0.125 \mathrm{~g} \mathrm{~kg}^{-1}$. In the JGOFS EqPac program we found that a more stringent definition ( $0.03 \mathrm{~g} \mathrm{~kg}^{-1}$ ) was needed to define the depth that had most recently undergone mixing based on the first break in slope of the density profile (Gardner et al., 1995). Brainerd and Gregg (1995) distinguished between the "mixed layer" (generally the top of the seasonal thermocline) and the "mixing layer" (the depth of most recent mixing), with the latter value sometimes requiring a change as small as 0.01 density units for accurate determination.

In EqPac we found that the mixed layer could change from $10 \mathrm{~m}$ to as much as $70 \mathrm{~m}$ on a diurnal scale. Data from a neutrally buoyant float developed by D'Asaro et al. (1996) demonstrated that parcels of water (and associated particles) actually move up and down through the mixing layer with a $1-$ to $4-h$ period in one data set from the northeast Pacific, with deep mixing at night and shallow mixing during the day (Fig. 4). This type of mixing was modeled by Woods and Onken (1982), in which they traced the path of a particle in the mixed layer. assuming that particles completed cycles through the mixed layer in $\sim 30$ min. Although they noted that particles will be isolated below the mixed layer during the day, they assumed that because the particles settled at only $2 \mathrm{~m} / \mathrm{d}$. these particles would be reincorporated into the mixed layer the next day, resulting in no loss of particles from the mixed layer by this mechanism. What they did not anticipate, however, is that aggregates can settle at a rate of 50-250 $\mathrm{m} / \mathrm{d}$ (Diercks and Asper, 1997). Thus, if an aggregate were mixed downward and isolated below the mixed layer, it has little chance of being reincorporated into the mixed layer the following day and is lost from the surface. Woods and Onken (1982) also concluded that the diurnal change in depth of the mixed layer would not cause a diurnal variation in the concentration of particles. Our results in the equatorial Pacific were quite different.

Instead of using models or a hightech, neutrally buoyant float. we took ad-

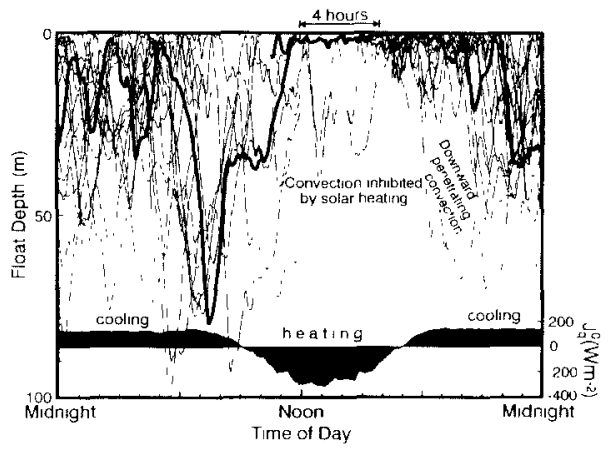

Fig. 4: Time-depth trajectory of a neatrally buovant float designed by D'Asaro et al. (1996) over several daily cycles superimposed on one daily plot showing deep mixing daring the night and shallow mixing during the day (after MoNeil and Farmer, 1995). These dynamics are important in the growth and export of phytoplankton in and from surface waters.

vantage of the billions and billions of "neutrally buoyant" floats that exist in surface waters as phytoplankton. Admittedly, phytoplankton aren't conservative; they can grow, divide, be consumed, aggregate, and settle out of the system. Nevertheless, their net abundance can be quantified adequately by measuring inherent optical properties such as beam attenuation (Chung et al., 1996). Repeated vertical profiles at the same location demonstrated the diurnal cycle in particle concentration (Gardner et al., 1995). A close examination of two diurnal cycles selected from a 2-wk time series on the equator reveals how small particles produced in surface waters during the day are mixed downward at a rate $(20-70 \mathrm{~m}$ $\left.\mathrm{d}^{-1}\right)$ much faster than their Stokesian setthing velocities (Fig. 5). Particle concentrations in surface waters increased during day 285 from $70 \mu \mathrm{g} \mathrm{l}$ ' at dawn to $110 \mu \mathrm{g} 1$ ' at dusk, when the mixing depth was only $20 \mathrm{~m}$. By dawn the next morning the surface-water particle concentration dropped from 110 to $75 \mu \mathrm{g} \mathrm{l}$ ', and the mixing depth was $70 \mathrm{~m}$.

The daytime increases in particle mass were due to primary production in excess of grazing. The nighttime decreases have been attributed to grazing. and although that was probably the dominant factor for reducing biomass in surface waters. there were other contributing factors. At dusk. the depth of mixing was $20 \mathrm{~m}$. over which particle concentrations were constant and below which 


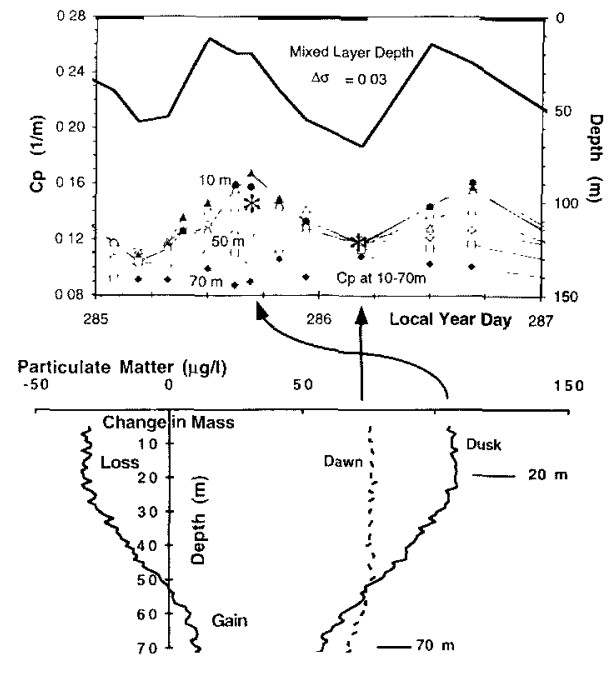

Fig. 5: Mixed layer depth (based on a $\Delta \sigma$ of $0.03 \mathrm{~kg} \mathrm{l}^{-1}$ ) and beam attenuation at $10,20,30,40,50,60$, and $70 \mathrm{~m}$ during $2 \mathrm{~d}$ on the equator at $140^{\circ} \mathrm{W}$. Divergent isopleths and a thin mixed laver indicate stratification and high particle production during the day. Convergent isopleths result from deep overturning that causes a thick mixed layer and carries particles downward at night. Profiles of particle mass at dusk and dawn demonstrate the loss of particle mass in surface waters and gain of particle mass below $50 \mathrm{~m}$ during the night as a result of grasing, particle loss, and deepening of the mixed laver.

they decreased with depth. At dawn the next morning, particle concentrations were much lower in the surface, but were higher below $50 \mathrm{~m}$. The deeper mixing during the night "pumped" highconcentration surface water downward and entrained water with lower concentrations into surface waters. At dawn. particle concentrations were uniform down to $50 \mathrm{~m}$, while the depth of mixing was $70 \mathrm{~m}$, suggesting that although turnover may have occurred to $70 \mathrm{~m}$, mixing was not complete. The calculated loss of particle mass in the upper $50 \mathrm{~m}$ was greater than the gain at depth. Most of this difference is due to grazing and remineralization. Another contributing factor is that when particles form large aggregates, they are less likely to be quantitatively detected in the small-volume beam of the transmissometer. Thus aggregation of particles could cause an apparent decrease in particle mass, even though the mass is still present. Of course, large aggregates are also more likely to settle than individual small par- ticles, resulting in a real decrease in mass.

Thus the mixed layer pump moves particles downward faster than they can settle by gravity. The same process can mix nutrient-rich deep water upward and fuel primary production the following day, and it can "pump" any component up or down that has a gradient across the diurnal depth excursion of the mixedlayer. Such mixing is much more rapid than diffusion. Similar conditions have been found to exist during some seasons and locations in the Indian Ocean during the JGOFS Arabian Sea program.

In summary, we must change our paradigm from the assumption that particle fluxes are dominated by large particles to a paradigm of fluxes being dominated by rapidly settling particles, whether they be small, dense fecal pellets or large, lowdensity aggregates. Aggregates can contribute significantly to the flux, but quantification is not always as simple as we would hope. The deficiency of ${ }^{234} \mathrm{Th}$ in surface waters may function as a proxy for the flux of carbon, but the method applies only in the upper $\sim 100 \mathrm{~m}$. Furthermore, although there may be a $\mathrm{C}^{234} \mathrm{Th}$ ratio to determine the carbon flux, there are no proposed ratios of foraminifera: ${ }^{234} \mathrm{Th}$, $\mathrm{Si}:{ }^{234} \mathrm{Th}$, or other biogeochemically important elements, so a sediment trap, used properly, is still an important tool for collecting particles.

Historically, we've progressed in studying particle fluxes from calculating settling rates of individual particles, through using sediment traps and developing radionuclide proxies, to measuring and modeling the whole carbon budget within a given system in the JGOFS and other programs. JGOFS is just entering its synthesis stage, so we can expect even better models in the future. In creating models, it is important to account for the mechanisms of particle production, exchange, and loss within a system. The dynamics of the mixed layer is important in this regard.

\section{Acknowledgments}

I thank Ann Gargett for her leadership in publishing this group of papers. Comments from Mary Jo Richardson were especially helpful. Many colleagues in JGOFS and related programs have provided data and insight over the years. Support for this work came from the National Science Foundation (Chemical Oceanography), the Office of Naval
Research, and the Department of Energy.

\section{References}

Bishop. J.K.B., R.W. Collier, D.R. Ketten and J.M. Edmond, 1980: The chemistry, biology, and vertical flux of particulate matter from the upper $1500 \mathrm{~m}$ of the Panama Basin in the Equatorial Pacific Ocean. Deep-Sea Res., 27A, 615-640.

Brainerd, K.E. and M.C. Gregg. 1995: Surface mixed layer and mixing layer depths. DeepSea Res, 42, 1521-1543.

Buesseler, K.O., J.K. Cochran, M.P. Bacon and H.D. Livingston, 1992: Carbon and nitrogen export during the JGOFS North Atlantic Bloom Experiment estimated from ${ }^{234} \mathrm{Th}^{228} \mathrm{U}$ disequilibria. Deep-Sea Res., 39, 1115-1137. A.F. Michael, D.A. Siegel, and A.H. Knap, 1994: A three-dimensional time-dependent approach to calibrating sediment trap fluxes. Global Biogeochemical Cycles, 8, 179-193. , J.A. Andrews, M.C. Hartman. R. Belastock and F. Chai, 1995: Regional estimates of the export flux of particulate organic carbon derived from thorium-234 during the JGOFS EqPac program. Deep-Sea Res., 42, $777-$ 804.

Chung. S.P., W.D. Gardner, M.J. Richardson. I.D. Walsh and M.R. Landry, 1996: Beam attenuation and microorganisms: spatial and temporal variations in small particles along $140^{\circ} \mathrm{W}$ during 1992 JGOFS-EqPac transects. Deep-Sea Res. II, 42, 1205-1226.

D'Asaro. E.A., D.M. Farmer, J.T. Osse and G.T. Dairiki, 1996: A Lagrangian Float. J. Atmos. Ocean. Tech., 13, 1230-1246.

Diercks, A.R. and V.L. Asper, 1997: In situ settling speeds of marine snow aggregates below the mixed layer-Black Sea and Gulf of Mexico. Deep-Sea Res., I, 44, 385-398.

Emerson, S., P.D. Quay, C. Stump, D. Wilbur and R. Schudlich, 1995: Chemical tracers of productivity and respiration in the subtropical Pacific Ocean. J. Geophys. Res., 100. 15873-15887.

Gardner, W.D., S.P. Chung, M.J. Richardson and I.D. Walsh, 1995: The oceanic mixed-layer pump. Deep-Sea Res. II, 42, 757-775. and I.D. Waish, 1990: Distribution of macroaggregates and fine-grained particles across a continental margin and their potential role in fluxes. Deep-Sea Res., 37 , 401-4I1.

Honjo, S., K.W. Doherty, Y.C. Agrawal and V.L. Asper, 1984: Direct optical assessment of large amorphous aggregates (marine snow) in the deep ocean. Deep-Sea Res.. 31, $67-76$.

and S.J. Manganini, 1993: Annual biogenic particle fluxes to the interior of the North Atlantic Ocean; studies at $34^{\circ} \mathrm{N} 21^{\circ} \mathrm{W}$ and $48^{\circ} \mathrm{N} 21^{\circ} \mathrm{W}$. Deep-Sea Res., 40, 587-607.

Karl, D.M., J.R. Christian, J.E. Dore. D.V. Hebel, R.M. Letelier. L.M. Tupas and C.D. Winn. 1996: Seasonal and interannual variability in primary production and particle flux at Station ALOHA. Deep-Sea Res., 43, 539-568.

Lal, D. and A. Lerman. 1973: Dissolution and behavior of particulate biogenic matter in the ocean: some theoretical calculations. J. Geophys. Res., 78, 7100-7111.

Levitus, S., 1982: Climatological Atlas of the 
World. Washington, DC: U.S. Dept. of Commerce, NOAA. NOAA Prof. Paper 13.

Martin, J.H., S.E. Fitzwater. R.M. Gordon. C.N. Hunter and S.J. Tanner, 1993: Iron, primary production and carbon-nitrogen flux studies during the JGOFS North Atlantic Bloom Experiment. Deep-Sea Res., 40, 115-134.

McCave. I.N., 1975: Vertical fiux of particles in the ocean. Deep-Sea Res., 22, 491-502.

McNeil. C.L. and D.M. Farmer, 1995: Observations of the influence of diurnal convection on upper ocean dissolved gas measurements. $J$. Mar. Res., 53, 151-169.

Michaels, A.F.. N.R. Bates, K.O. Buesseler, C.A. Carlson and A.H. Knap, 1994: Carbon system imbalances in the Sargasso Sea. Nature,

\section{$372,537-540$.}

Murray. J.W.. J. Young. J. Newton. J. Dunne. T. Chapin, B. Paul and J.J. McCarthy, 1996: Export flux of particulate organic carbon from the central equatorial Pacific determined using a combined drifting trap- ${ }^{23+} \mathrm{Th}$ approach. Deep-Sea Res. II, 4I, 10951132.

Shanks, A.L. and J.D. Trent. 1980: Marine snow: sinking rates and potential role in vertical flux. Deep-Sea Res., 27A, 137-14.3.

Steinberg. D.K., C.A. Carlson, N.R. Bates and A.F. Michaels. 1997: Zooplankton vertical migrations and the active transport of dissolved organic and inorganic carbon in the Sargasso Sea. American Society of Limnol- ogy and Oceunography, Program and Ahstracts, 311.

U.S. GOFS Report No. 10, 1989: Sediment Trap Technology and Sampling. Available from U.S. JGOFS Planning Office, Woods Hole Oceanographic Institution. Woods Hole. MA. 94 pp.

Walsh, I.D. and W.D. Gardner. 1994: A model of aggregate appearance and loss during the spring bloom in the North Atlantic. EOS, Transactions of the American Geophswical Union, v. 75:47.

Woods. J.D. and R. Onken, 1982: Diurnal variation and primary production in the ocean-preliminary results of a Lagrangian ensemble model. J. Plankton Re's. 4. 735-756. 